\title{
Mid-term Clinical Outcomes of Immediate Protamine Use Following Elective Percutaneous Coronary Interventions
}

\author{
Masayuki Kubota, ${ }^{1}$ MD, Kenichi Sakakura, ${ }^{1}$ MD, Kei Yamamoto, ${ }^{1}$ MD, Yousuke Taniguchi, ${ }^{1}$ MD, \\ Takunori Tsukui, ${ }^{1}$ MD, Masaru Seguchi, ${ }^{1}$ MD, Hiroshi Wada, ${ }^{1}$ MD, \\ Shin-ichi Momomura, ${ }^{1}$ MD and Hideo Fujita, ${ }^{1}$ MD
}

\begin{abstract}
Summary
Bleeding complication has been considered as a serious problem in current percutaneous coronary interventions (PCI). Fortunately, several groups have already reported the effectiveness of protamine use just after PCI to immediately remove any arterial sheath. However, there is a concern that protamine reversal may increase non-occlusive thrombus and, in turn, lead to mid-term cardiovascular events such as target vessel revascularization (TVR) or stent thrombosis. Thus, the purpose of this study was to evaluate whether protamine use following elective PCI was associated with mid-term clinical outcomes. In total, 472 patients were included in this study; subsequently, they were divided into protamine group $(n=142)$ and non-protamine group $(n=330)$. The primary endpoint was the composite of ischemia-driven TVR and stent thrombosis. The median follow-up period was determined to be at 562 days. In total, 32 primary endpoints were observed during the study period, and the incidence of primary endpoints tended to be greater in the protamine group than in the non-protamine group $(P=0.056)$. However, the lesion length, the degree of calcification, and the prevalence of hemodialysis were significantly determined greater in the protamine group than in the non-protamine group. In the multivariate Cox proportional hazards model, the use of protamine (versus non-protamine: hazard ratio 0.542 and $95 \%$ confidence interval $0.217-1.355, P=0.191$ ) was deemed not to be associated with the primary endpoint after controlling legion length, calcification, and hemodialysis. In conclusion, immediate protamine use following elective PCI did not increase mid-term ischemia-driven TVR or stent thrombosis. However, immediate protamine use after PCI should be discussed further for the safety of the patient.
\end{abstract}

Key words: Bleeding complication, Target vessel revascularization, Stent thrombosis

(Int Heart J 2020; 61: 865-871)

A lthough percutaneous coronary interventions (PCI) have advanced in decades, bleeding complication has been identified as a serious concern in current PCI. ${ }^{1-3)}$ Although arterial closure devices such as Perclose ProGlide (Abbott Vascular, Abbott Park, IL) were used for earlier hemostasis and shorter ambulation, these devices might not reduce major vascular complications when compared to manual compression., ${ }^{4,5)}$ Thus, manual compression is still required and deemed important even after the emergence of arterial closure devices.

The prevention of major bleeding and vascular complications following PCI has already been widely discussed. ${ }^{6}$ Several groups have proven the effectiveness of protamine use just after PCI in removing any arterial sheath in the bare-metal stent era. ${ }^{7-9)}$ We also reported that protamine use just after PCI did not provoke acute stent thrombosis. ${ }^{10)}$ However, a concern has been raised regarding protamine use immediately after PCI as it may increase non-occlusive thrombus formation within a stent, even if it does not result in acute stent occlusion. Further- more, immediate protamine use may lead to mid-term cardiovascular events such as target vessel revascularization (TVR) or stent thrombosis, partly because an angioscopic study showed a significant association of subclinical intrastent thrombus detected 9 months after implantation with future target lesion revascularization. ${ }^{11)}$ The purpose of this study was to determine the association between protamine use following elective PCI and mid-term clinical outcomes.

\section{Methods}

Study lesions: We identified consecutive 849 patients who underwent PCI in the Saitama Medical Center, Jichi Medical University, from April 2017 to March 2018. The exclusion criteria were as follows: (1) emergent PCI to the culprit of acute coronary syndrome (ACS), (2) unsuccessful PCI, (3) $\geq 2$ vessels were treated simultaneously in one session, (4) PCI to the lesion of coronary artery bypass graft, and (5) argatroban use because of the history

From the ${ }^{1}$ Division of Cardiovascular Medicine, Saitama Medical Center, Jichi Medical University, Saitama, Japan.

Address for correspondence: Kenichi Sakakura, MD, Division of Cardiovascular Medicine, Saitama Medical Center, Jichi Medical University, 1-847, Amanuma, Omiya-ku, Saitama, 330-8503, Japan. E-mail: ksakakura@jichi.ac.jp

Received for publication February 23, 2020. Revised and accepted May 1, 2020

Released in advance online on J-STAGE September 12, 2020.

doi: $10.1536 /$ ihj.20-126

All rights reserved by the International Heart Journal Association. 
of heparin-induced thrombocytopenia. In addition, in case a patient underwent multiple PCI during this study period, only the first PCI was included. The final study population was subsequently divided into protamine group and non-protamine group, according to the use of protamine just after PCI. The primary endpoint of this study was the composite of ischemia-driven TVR and stent thrombosis. We defined the day of PCI as the index day (day 1). Patients were followed until the last follow-up date (September 31, 2019). This study was approved by the Institutional Review Board of Saitama Medical Center, Jichi Medical University, and written informed consent was waived because of the retrospective nature of the study.

Elective PCI procedures: Elective PCI was performed using standard techniques via the radial artery, femoral artery, or, rarely, brachial artery. We preferred $\geq 7$ Fr system for complex PCI such as PCI to chronic total occlusion or rotational atherectomy. We also used the additional femoral vein sheath in case of complex or high-risk lesions such as chronic total occlusion or severe calcified lesions that required rotational atherectomy, even if PCI was performed via radial artery. Although the radial artery as well as the femoral artery could be used for $7 \mathrm{Fr}$ system, only the femoral artery was used for $8 \mathrm{Fr}$ system. The choice of PCI devices such as guidewires, balloons, and stents was left to the discretion of the interventional cardiologists at our cardiology center. Activated clotting time (ACT) was maintained as $>250$ seconds during PCI procedures. An IVUS was routinely used for almost all lesions. The protamine was then administered in most cases of trans-femoral or trans-brachial PCI. The protamine was sometimes administered in cases of additional femoral vein sheath. Furthermore, there were a few cases in which the protamine was administered because of ACT prolongation. In the protamine group, manual compression was mostly performed in the catheterization laboratory just after slow injection of protamine. We made a dilution of protamine in saline (total $20 \mathrm{~mL}$ ) and then injected intravenously in approximately 5 minutes. Although our target ACT was $<200$ seconds for manual compression, ACT was not routinely measured after the administration of protamine. On the other hand, some cases of trans-femoral or trans-brachial cases did not receive protamine in the catheter laboratory, for example, patients whose sheath was not removed at the catheter laboratory or patients whose sheath was removed using a closure device.

Definition: Hypertension was defined as systolic blood pressure > $140 \mathrm{mmHg}$, diastolic blood pressure > 90 $\mathrm{mmHg}$, or medical treatment for hypertension. ${ }^{10)}$ Meanwhile, dyslipidemia was defined as a total cholesterol level $\geq 220 \mathrm{mg} / \mathrm{dL}$, or a low-density lipoprotein cholesterol level $\geq 140 \mathrm{mg} / \mathrm{dL}$, or medical treatment for dyslipidemia or a history of dyslipidemia. ${ }^{12)}$ Diabetes mellitus was defined as hemoglobin A1c value $\geq 6.5 \%$ (NGSP) or medical treatment for diabetes mellitus. ${ }^{12,13)}$ We have calculated the estimated glomerular filtration rate (eGFR) from the serum creatinine level, age, weight, and gender using the following formula as follows: eGFR $=194 \times$ $\mathrm{Cr}^{-1.094} \times$ age $^{-0.287}$ (male), eGFR $=194 \times \mathrm{Cr}^{-1.094} \times \mathrm{age}^{-0.287} \times$ 0.739 (female). ${ }^{14)}$ Chronic kidney disease was defined as the eGFR $<60 \mathrm{~mL} /$ minute $/ 1.73 \mathrm{~m}^{2}$. Quantitative coronary angiography parameters were measured using a cardiovascular angiography analysis system (QAngio XA 7.3, MEDIS Imaging Systems, Leiden, Netherlands). Calcification was then identified as readily apparent radiopacities within the vascular wall at the site of the stenosis and was further classified as none/mild, moderate, and severe. ${ }^{15}$ ) Chronic total occlusion was defined as total occlusion with complete interruption of antegrade blood flow as assessed by coronary arteriography and with an estimated duration of occlusion of $\geq 3$ months. ${ }^{16)}$ Stent thrombosis was defined according to the ARC consensus, and classified as definite, probable, and possible. ${ }^{17,18)}$ In this study, the case of "definite" or "probable" was included as stent thrombosis.

Statistical analysis: Data are presented as the mean \pm standard deviation or percentage. Categorical variables are presented as numbers (percentage) and compared with Pearson's chi-squared test or Fisher's exact test. The Shapiro-Wilk test was also performed in order to determine if the continuous variables were normally distributed. The unpaired Student's $t$-test was used for normally distributed continuous variables, while the Mann-Whitney $U$ test was used for non-normally distributed continuous variables. The event-free survival curves were drawn using Kaplan-Meier methods, and the log-rank test was used in calculating statistical differences between the protamine and non-protamine groups. The multivariate Cox regression analysis was also conducted to investigate whether the primary endpoint was statistically significant between the protamine and non-protamine groups after controlling confounding factors. In this model, the primary endpoint (the composite of stent thrombosis and ischemia-driven TVR) was used as the dependent variable. The use of protamine and several clinical factors were adopted as independent variables. Only three or four variables could be used as independent variables, because the number of events (the composite of ischemia-driven TVR and stent thrombosis) was 32. Independent variables were chosen according to the relevance for the primary endpoint. Since the association between the lesion complexity and the incidence of cardiovascular events was widely reported, ${ }^{19,20)}$ legion length and calcification were selected as independent variables. ${ }^{21,22)}$ Furthermore, the association between TVR and hemodialysis was also well known; ${ }^{23,24}$ chronic renal failure on hemodialysis was also selected as an independent variable. All variables were simultaneously adjusted in one step on multivariate Cox hazard analysis. The hazard ratio (HR) and the $95 \%$ confidence interval (CI) were also calculated. A $P$-value $<0.05$ was considered statistically significant. We analyzed all data using SPSS ver. 24 for Windows (SPSS, Inc., Chicago, Illinois).

\section{Results}

In our medical center, 849 patients were identified to have underwent elective PCI during the study period. We excluded 377 patients that fell under the exclusion criteria. In total, 472 patients were included as the final study population, and they were subsequently divided into protamine group $(n=142)$ and the non-protamine group $(n=$ 330). The study flow chart is shown in Figure 1. 


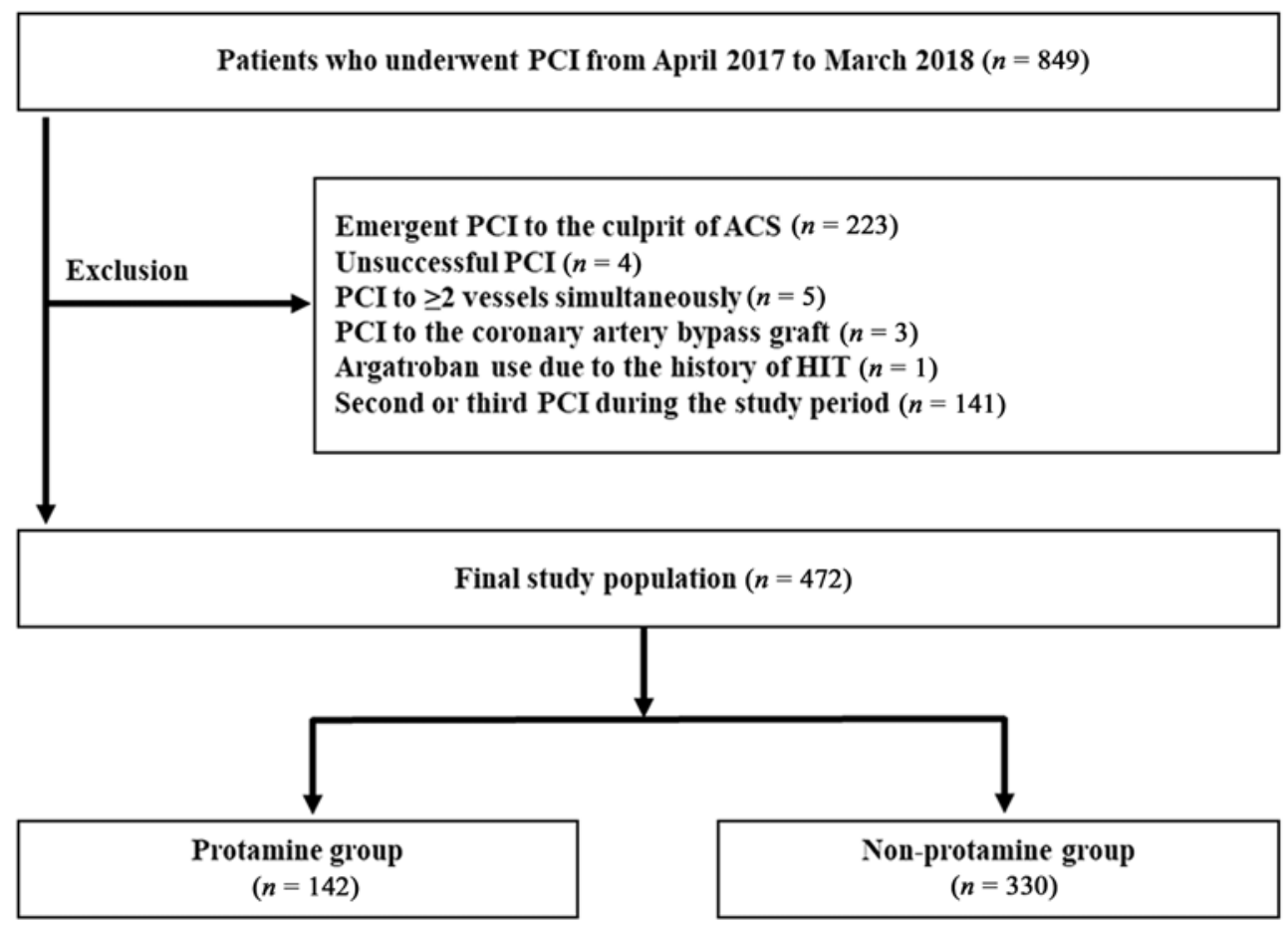

Figure 1. Study flowchart. PCI indicates percutaneous coronary intervention; ACS, acute coronary syndrome; and HIT, heparin-induced thrombocytopenia.

Table I shows the comparison of clinical characteristics between the two groups. Most of clinical characteristics were found to be comparable between the two groups. Furthermore, the prevalence of hemodialysis was significantly greater in the protamine group $(28.9 \%)$ than the non-protamine group $(2.4 \%)(P<0.001)$. Table II shows the comparison of lesion and procedural characteristics between the two groups. Trans-radial PCI was more frequently performed in the non-protamine group than in the protamine group $(P<0.001)$. The legion length was determined to be longer in the protamine group $(25.4 \pm 19.9$ $\mathrm{mm})$ than in the non-protamine group $(19.1 \pm 12.2 \mathrm{~mm})$ $(P=0.007)$. The degree of calcification was more severe in the protamine group than in the non-protamine group $(P<0.001)$.

Kaplan-Meier curves were constructed to compare the primary endpoint, ischemia-driven TVR, and stent thrombosis between the two groups in Figure 2. The median follow-up duration was 562 days (Q1: 364 days, Q3: 699 days). In total, 32 primary endpoints (15 events in the protamine group and 17 events in the non-protamine group) were observed during the study period. The incidence of primary endpoints tended to be greater in the protamine group than in the non-protamine group $(P=$ 0.056). In total, 31 ischemia-driven TVR (14 events in the protamine group and 17 events in the non-protamine group) were observed during the study period. The incidence of ischemia-driven TVR tended to be greater in the protamine group than in the non-protamine group $(P=$ 0.098). There was no probable stent thrombosis. Only two definite stent thromboses (one in the protamine group and one in the non-protamine group) were observed during the study period. A definite stent thrombosis in the protamine group occurred at 24 days following zotarolimus-eluting stent implantation to a non-AMI lesion. A definite stent thrombosis in the non-protamine group occurred at 472 days following everolimus-eluting implantation to a nonAMI lesion. In regard to the bleeding complications, the incidence of gastrointestinal bleeding was comparable between the protamine group $(2.8 \%)$ and the non-protamine group $(0.9 \%)$ during the study period $(P=0.206)$. Furthermore, the incidence of cerebral hemorrhage was also comparable between the protamine group $(2.8 \%)$ and the non-protamine group $(0.9 \%)$ during the study period $(P=$ 0.206). The multivariate Cox hazard model, which investigates the association between the use of protamine and the primary endpoint, is shown in Table III. The use of protamine was not associated with the primary endpoint after controlling legion length, moderate to severe calcification, and hemodialysis (versus non-protamine use: HR $0.542,95 \%$ CI $0.217-1.355, P=0.191)$.

\section{Discussion}

Although the incidence of primary endpoint tended to be greater in the protamine group compared to that in the non-protamine group in an unadjusted model, the incidence of primary endpoint was not associated with the use of protamine after controlling confounding factors. These results may reduce the concern that use of protamine just after PCI will lead to mid-term cardiovascular events such as TVR or stent thrombosis and further facilitate the use of protamine for patient's safety.

Protamine use tended to be associated with worse 
Table I. Comparison of Clinical Characteristics Between the Protamine Group and the Non-protamine Group

\begin{tabular}{|c|c|c|c|c|}
\hline & $\begin{array}{c}\text { All } \\
(n=472)\end{array}$ & $\begin{array}{l}\text { Protamine group } \\
\quad(n=142)\end{array}$ & $\begin{array}{l}\text { Non-protamine group } \\
(n=330)\end{array}$ & $P$-value \\
\hline Age, year & $71.0 \pm 10.1$ & $71.8 \pm 9.6$ & $70.6 \pm 10.3$ & 0.273 \\
\hline Male sex, $n(\%)$ & $384(81.4)$ & $107(75.4)$ & $277(83.9)$ & 0.039 \\
\hline Overweight (BMI $\left.\geq 25 \mathrm{~kg} / \mathrm{m}^{2}\right), n(\%)$ & $171(36.2)$ & $46(32.4)$ & $125(37.9)$ & 0.297 \\
\hline Hypertension, $n(\%)$ & $437(92.6)$ & $135(95.1)$ & $302(91.5)$ & 0.250 \\
\hline Diabetes mellitus, $n(\%)$ & $229(48.5)$ & $70(49.3)$ & $159(48.2)$ & 0.841 \\
\hline Diabetes mellitus using insulin, $n(\%)$ & $40(8.5)$ & $17(12.0)$ & $23(7.0)$ & 0.103 \\
\hline Dyslipidemia, $n(\%)$ & $418(88.6)$ & $124(87.3)$ & $294(89.1)$ & 0.637 \\
\hline Current smoker, $n(\%)$ & $113 / 471(24.0)$ & $22(15.6)$ & $91(27.6)$ & 0.005 \\
\hline Chronic kidney disease, $n(\%)$ & $226(47.9)$ & $87(61.3)$ & $139(42.1)$ & $<0.001$ \\
\hline Hemodialysis, $n(\%)$ & $49(10.4)$ & $41(28.9)$ & $8(2.4)$ & $<0.001$ \\
\hline History of previous myocardial infarction, $n(\%)$ & $193(40.9)$ & $66(46.5)$ & $127(38.5)$ & 0.126 \\
\hline History of previous CABG, $n(\%)$ & $20(42.4)$ & $11(7.7)$ & $9(2.7)$ & 0.022 \\
\hline History of previous PCI, $n(\%)$ & $226(47.9)$ & $76(53.5)$ & $150(45.5)$ & 0.110 \\
\hline Serum creatinine, $\mathrm{mg} / \mathrm{dL}$ & $1.67 \pm 2.29$ & $3.06 \pm 3.49$ & $1.07 \pm 1.05$ & $<0.001$ \\
\hline $\mathrm{eGFR}, \mathrm{mL} / \mathrm{minute} / 1.73 \mathrm{~m}^{2}$ & $59.2 \pm 26.2$ & $46.1 \pm 31.4$ & $64.8 \pm 21.4$ & $<0.001$ \\
\hline Low density lipoprotein, mg/dl $(n)$ & $92.0 \pm 31.0(n=465)$ & $84.5 \pm 27.8(n=139)$ & $95.2 \pm 31.8(n=326)$ & 0.001 \\
\hline Hemoglobin, g/dL & $12.8 \pm 1.8$ & $12.4 \pm 1.7$ & $13.0 \pm 1.8$ & 0.001 \\
\hline Prothrombin time-INR, $(n)$ & $1.06 \pm 0.15(n=455)$ & $1.05 \pm 0.13(n=137)$ & $1.06 \pm 0.16(n=318)$ & 0.676 \\
\hline Dual antiplatelet therapy, $n(\%)$ & & & & 0.753 \\
\hline Aspirin and clopidogrel, $n(\%)$ & $318(67.4)$ & $98(69.0)$ & $220(66.7)$ & \\
\hline Aspirin and prasugrel, $n(\%)$ & $146(30.9)$ & $41(28.9)$ & $105(31.8)$ & \\
\hline Other combination, $n(\%)$ & $8(1.7)$ & $3(2.1)$ & $5(1.5)$ & \\
\hline Anticoagulation, $n(\%)$ & & & & 0.018 \\
\hline Direct oral anticoagulant, $n(\%)$ & $38(8.1)$ & $4(2.8)$ & $34(10.3)$ & \\
\hline Warfarin, $n(\%)$ & $13(2.8)$ & $3(2.1)$ & $10(3.0)$ & \\
\hline No anticoagulation, $n(\%)$ & $421(89.2)$ & $135(95.1)$ & $286(86.7)$ & \\
\hline
\end{tabular}

Data were expressed as mean \pm SD or numbers (percentages). A Student's $t$-test was used for normally distributed continuous variables, and Mann-Whitney $U$ test was used for abnormally distributed continuous variables. A chi-square test was used for categorical variables. BMI indicates body mass index; CABG, coronary artery bypass grafting; PCI, percutaneous coronary intervention; eGFR, estimated glomerular filtration rate; and INR, international normalized ratio.

clinical outcomes in Kaplan-Meier curves, but was not associated with mid-term clinical outcomes after controlling lesion length, moderate to severe calcification, and hemodialysis. In our catheter laboratory, protamine was commonly used in trans-femoral or trans-brachial PCI, whereas protamine was rarely used in trans-radial PCI except for cases that required an additional femoral puncture for venous sheath or IABP. Since non-complex PCI was usually performed via radial artery, PCI that required protamine might be more complex as compared to PCI that did not use protamine. In fact, lesion length was significantly shorter in the non-protamine group than in the protamine group, and degree of calcification was determined to be greater in the protamine group than in the nonprotamine group. Moreover, the prevalence of hemodialysis was also found higher in the protamine group than in the non-protamine group. Because the association between the complexity of PCI and the incidence of cardiovascular events has been widely reported, ${ }^{19,20)}$ the lesion complexity should be the strong confounding factor in unadjusted Kaplan-Meier curves. Furthermore, TVR following PCI is more frequently observed in patients with hemodialysis than in patients without hemodialysis. ${ }^{23,24}$ Therefore, it was reasonable that the association between protamine use and adverse outcomes were not significant after controlling lesion length, calcification, and hemodialysis.

We should also discuss why protamine use did not increase the ischemia-driven TVR or stent thrombosis. We hypothesized that using protamine immediately after PCI may increase non-occlusive thrombus formation within a stent, because several preclinical studies using current drug-eluting stents showed acute thrombogenicity in spite of sufficient blood flow. ${ }^{25,26)}$ Otsuka, et al. reported that only 1 hour of circulating blood with insufficient ACT (150 seconds to 190 seconds) would result in plateletmediated thrombus formation in a swine shunt model. ${ }^{25}$ However, their swine did not have any antiplatelet therapy. Therefore, the main reason underlying our results would be the fact that dual antiplatelet therapy was already administered before elective PCI. Unlike emergent PCI, the antiplatelet effect would be stable at the time of elective PCI. Therefore, as long as the catheter devices such as guidewires, balloons, and guide catheters were removed, the anticoagulation effect by heparin would not be necessary. Another reason may pertain to the exclusion criteria regarding emergent PCI to the culprit of ACS. The culprit of ACS would have the residual thrombus, ${ }^{27)}$ which might require continuous anticoagulation by heparin. On the other hand, it is more important to prevent bleeding complications in primary PCI for ST-elevation myocardial infarction (STEMI), because bleeding complications in patients with STEMI were closely associated with inhospital death. ${ }^{28)}$ Since we did not use protamine in the catheter laboratory in cases of emergent PCI, we could not evaluate the risk and benefit of protamine in cases of emergent PCI. Further studies are warranted to examine 
Table II. Comparison of Lesion and Procedural Characteristics Between the Protamine Group and the Non-protamine Group

\begin{tabular}{|c|c|c|c|c|}
\hline & $\begin{array}{c}\text { All } \\
(n=472)\end{array}$ & $\begin{array}{l}\text { Protamine group } \\
\qquad(n=142)\end{array}$ & $\begin{array}{l}\text { Non-protamine group } \\
\quad(n=330)\end{array}$ & $P$-value \\
\hline Diagnosis & & & & 0.394 \\
\hline PCI to the culprit of non-AMI, $n(\%)$ & $403(85.4)$ & $118(83.1)$ & $285(86.4)$ & \\
\hline PCI to the culprit of AMI, $n(\%)$ & $69(14.6)$ & $24(16.9)$ & $45(13.6)$ & \\
\hline Target coronary artery & & & & 0.342 \\
\hline Left main trunk-left anterior descending artery, $n(\%)$ & $237(50.2)$ & $72(50.7)$ & $165(50.0)$ & \\
\hline Left circumflex artery, $n(\%)$ & $73(15.5)$ & $17(12.0)$ & $56(17.0)$ & \\
\hline Right coronary artery, $n(\%)$ & $162(34.3)$ & $53(37.3)$ & $109(33.0)$ & \\
\hline Number of narrowed coronary arteries & & & & 0.487 \\
\hline 1 & $271(57.4)$ & $77(54.2)$ & $194(58.8)$ & \\
\hline 2 & $152(32.2)$ & $47(33.1)$ & $105(31.8)$ & \\
\hline 3 & $49(10.4)$ & $18(12.7)$ & $31(9.4)$ & \\
\hline Final PCI procedures & & & & 0.512 \\
\hline Bare-metal stent implantation, $n(\%)$ & $15(3.2)$ & $4(2.8)$ & $11(3.3)$ & \\
\hline Drug-eluting stent implantation, $n(\%)$ & $404(85.6)$ & $121(85.2)$ & $283(85.8)$ & \\
\hline Drug-coated balloon angioplasty, $n(\%)$ & $49(10.4)$ & $17(12.0)$ & $32(9.7)$ & \\
\hline Percutaneous old balloon angioplasty, $n(\%)$ & $4(0.8)$ & $0(0)$ & $4(1.2)$ & \\
\hline Calcification & & & & $<0.001$ \\
\hline None or mild & $318(67.4)$ & $66(46.5)$ & $252(76.4)$ & \\
\hline Moderate & $41(8.7)$ & $19(13.4)$ & $22(6.7)$ & \\
\hline Severe & $113(23.9)$ & $57(40.1)$ & $56(17.0)$ & \\
\hline Reference diameter, mm & $2.52 \pm 0.67$ & $2.45 \pm 0.61$ & $2.56 \pm 0.69$ & 0.160 \\
\hline Legion length, mm & $21.0 \pm 15.2$ & $25.4 \pm 19.9$ & $19.1 \pm 12.2$ & 0.007 \\
\hline Puncture site for PCI & & & & $<0.001$ \\
\hline Radial artery, $n(\%)$ & $311(65.9)$ & $18(12.7)$ & $293(88.8)$ & \\
\hline Brachial artery, $n(\%)$ & $19(4.0)$ & $16(11.3)$ & $3(0.9)$ & \\
\hline Femoral artery, $n(\%)$ & $142(30.1)$ & $108(76.1)$ & $34(10.3)$ & \\
\hline Additional femoral puncture, $n(\%)$ & $110(23.3)$ & $67(47.2)$ & $43(13.0)$ & $<0.001$ \\
\hline Guiding catheter size & & & & $<0.001$ \\
\hline $5 \mathrm{Fr}, n(\%)$ & $1(0.2)$ & $1(0.7)$ & $0(0)$ & \\
\hline $6 \mathrm{Fr}, n(\%)$ & $253(53.6)$ & $38(26.8)$ & $215(65.2)$ & \\
\hline $7 \mathrm{Fr}, n(\%)$ & $203(43.0)$ & $93(65.5)$ & $110(33.3)$ & \\
\hline $8 \mathrm{Fr}, n(\%)$ & $15(3.2)$ & $10(7.0)$ & $5(1.5)$ & \\
\hline Rotational atherectomy, $n(\%)$ & $57(12.1)$ & $34(23.9)$ & $23(7.0)$ & $<0.001$ \\
\hline PCI for chronic total occlusion, $n(\%)$ & $15(3.2)$ & $10(7.0)$ & $5(1.5)$ & 0.003 \\
\hline Intra-aortic balloon pump support, $n(\%)$ & $6(1.3)$ & $4(2.8)$ & $2(0.6)$ & 0.070 \\
\hline Use of closure device, $n(\%)$ & $34(7.2)$ & $10(7.0)$ & $24(7.3)$ & 1.000 \\
\hline Heparin dose, unit & $6600 \pm 1470$ & $6845 \pm 1715$ & $6494 \pm 1340$ & 0.087 \\
\hline Heparin per body weight, unit/kg & $105 \pm 20$ & $112 \pm 23$ & $102 \pm 17$ & $<0.001$ \\
\hline Activated clotting time at end of PCI, second $(n)$ & $299 \pm 51(n=148)$ & $295 \pm 52(n=122)$ & $315 \pm 43(n=26)$ & 0.067 \\
\hline Protamine dose, $\mathrm{mg}$ & & $26 \pm 7$ & & \\
\hline \multicolumn{5}{|l|}{ Immediate side effects of protamine } \\
\hline Transient hypotension requiring vasopressor, $n(\%)$ & & $2(1.4)$ & & \\
\hline Anaphylaxis, $n(\%)$ & & 0 & & \\
\hline Skin rash, $n(\%)$ & & $3(2.1)$ & & \\
\hline \multicolumn{5}{|l|}{ Puncture site-related complications } \\
\hline Pseudoaneurysm, $n(\%)$ & $3(0.6)$ & $3(2.1)$ & 0 & 0.027 \\
\hline Bleeding requiring blood transfusion, $n(\%)$ & 0 & 0 & 0 & - \\
\hline
\end{tabular}

Data were expressed as mean \pm SD or numbers (percentages). A Student's $t$-test was used for normally distributed continuous variables, and Mann-Whitney $U$ test was used for abnormally distributed continuous variables. A chi-square test was used for categorical variables. PCI indicates percutaneous coronary intervention; and AMI, acute myocardial infarction.

the safety of protamine use in emergent PCI.

Clinical implications of this present study should be noted. Early studies, which reported protamine use just after PCI, focused on the safety in the acute phase. As compared to the protamine use just after PCI, there would be a greater risk of blood leakage through the side of the indwelling arterial sheath in the conventional manual procedure of hemostasis monitoring ACT. Indeed, our group reported that the use of protamine was inversely associated with bleeding requiring blood transfusion in trans-femoral PCI. ${ }^{10)}$ Thus, the advantage of protamine use was considered to enhance the safety in the acute phase. As this present study focused on the safety in the chronic phase, the safety of protamine use just after PCI was confirmed in a complementary way. Although the arterial closure devices were useful in trans-femoral PCI, fatal complications were also reported. ${ }^{4,5}$ Furthermore, the arterial closure devices were not available for trans-brachial PCI, which had more 

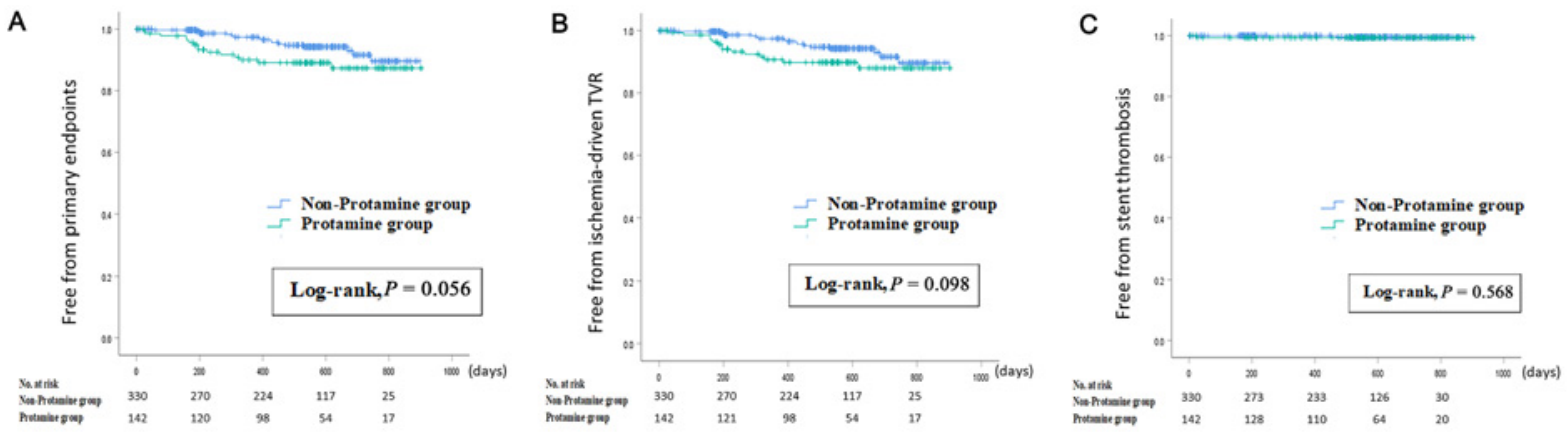

Figure 2. Kaplan-Meier survival curve between the protamine group and the non-protamine group. A: Comparison of the primary endpoint between the two groups. B: Comparison of ischemia-driven TVR between the two groups. C: Comparison of stent thrombosis between the two groups.

Table III. Multivariate Cox Hazard Model to Find the Association Between Protamine Use and Mid-term Clinical Outcomes

\begin{tabular}{|c|c|c|c|}
\hline \multicolumn{4}{|c|}{ Dependent variable: the composite of ischemia-driven TVR and stent thrombosis } \\
\hline Variables & Hazard ratio & $95 \%$ confidence interval & $P$-value \\
\hline Protamine use & 0.542 & $0.217-1.355$ & 0.191 \\
\hline Legion length (every $5.0-\mathrm{mm}$ increase) & 1.009 & $0.913-1.114$ & 0.865 \\
\hline Moderate to severe calcification (versus none or mild) & 2.428 & $1.120-5.259$ & 0.025 \\
\hline Hemodialysis & 8.912 & $3.642-21.807$ & $<0.001$ \\
\hline
\end{tabular}

All variables were simultaneously adjusted in one step.

vascular complications as compared to trans-femoral PCI. ${ }^{29)}$ The strategy of immediate protamine use after PCI should be more discussed further for the safety of the patients.

Study limitations: This study has several limitations. First, since this study was a single-center and retrospective study, there might be a risk of selection bias. Second, although the study population was not small, we did not perform power analysis for sample size calculation. Thus, there might be a risk of beta error. Third, we could not enter all potential confounding factors into the multivariate Cox regression analysis, because the number of the primary endpoint was only 32 . Since the number of events per variable should be less than $10,^{30,31)}$ we could include only additional three variables such as legion length, calcification, and hemodialysis. Fourth, although we focused on the safety in the chronic phase in the present study, we have to mention that a few immediate side effects of protamine such as transient hypotension or skin rash were observed in the protamine group. Fifth, although the incidence of pseudoaneurysm was significantly greater in the protamine group than in the non-protamine group, this difference would pertain to the higher incidence of femo$\mathrm{ral} / \mathrm{brachial}$ artery puncture in the protamine group.

\section{Conclusions}

The immediate use of protamine following elective PCI was determined to not increase mid-term ischemiadriven TVR or stent thrombosis. The immediate protamine use after PCI should be further examined for better patient's safety.

\section{Acknowledgments}

The authors acknowledge all staff in the catheter laboratory in Saitama Medical Center, Jichi Medical University for their technical support in this study.

\section{Disclosure}

Conflicts of interest: Dr. Sakakura has received speaking honoraria from Abbott Vascular, Boston Scientific, Medtronic Cardiovascular, Terumo, OrbusNeich, Japan Lifeline, and NIPRO; he has served as a proctor for Rotablator for Boston Scientific; and he has served as a consultant for Abbott Vascular and Boston Scientific. Prof. Fujita has served as a consultant for Mehergen Group Holdings, Inc.

\section{References}

1. Ali ZA, Poludasu S, Qureshi YH, et al. Impact of major bleeding on long-term mortality in anemic versus nonanemic patients undergoing percutaneous coronary intervention using bivalirudin. Am J Cardiol 2014; 113: 1481-6.

2. Ohno Y, Maekawa Y, Miyata $\mathrm{H}$, et al. Impact of periprocedural bleeding on incidence of contrast-induced acute kidney injury in patients treated with percutaneous coronary intervention. J Am Coll Cardiol 2013; 62: 1260-6.

3. Gayed M, Yadak N, Qamhia W, Daralammouri Y, Ohlow MA. Comorbidities and Complications in Nonagenarians Undergoing Coronary Angiography and Intervention. Int Heart J 2017; 58: 180-4.

4. Das R, Ahmed K, Athanasiou T, Morgan RA, Belli AM. Arterial closure devices versus manual compression for femoral haemostasis in interventional radiological procedures: a systematic re- 
view and meta-analysis. Cardiovasc Intervent Radiol 2011; 34: 723-38.

5. Martin JL, Pratsos A, Magargee E, et al. A randomized trial comparing compression, Perclose Proglide and Angio-Seal VIP for arterial closure following percutaneous coronary intervention: the CAP trial. Catheter Cardiovasc Interv 2008; 71: 1-5.

6. Baker NC, Ansel GM, Rao SV, et al. The choice of arterial access for percutaneous coronary intervention and its impact on outcome: An expert opinion perspective. Am Heart J 2015; 170: 13-22.

7. Pan M, Suarez de Lezo J, Medina A, et al. In-laboratory removal of femoral sheath following protamine administration in patients having intracoronary stent implantation. Am J Cardiol 1997; 80: 1336-8.

8. Ducas J, Chan MC, Miller A, Kashour T. Immediate protamine administration and sheath removal following percutaneous coronary intervention: a prospective study of 429 patients. Catheter Cardiovasc Interv 2002; 56: 196-9.

9. Thuesen L, Andersen HR, Botker HE, Dalby Kristensen S, Krusell LR, Lassen JF. In-laboratory femoral sheath removal after heparin reversal by protamine after percutaneous coronary intervention. EuroIntervention 2005; 1: 66-9.

10. Yamamoto S, Sakakura K, Taniguchi Y, et al. Safety of Reversing Anticoagulation by Protamine Following Elective Transfemoral Percutaneous Coronary Intervention in the Drug-Eluting Stent Era. Int Heart J 2018; 59: 482-8.

11. Okuno S, Ishihara T, Iida O, et al. Association of Subclinical Intrastent Thrombus Detected 9 Months After Implantation of 2 nd-Generation Drug-Eluting Stent With Future Major Adverse Cardiac Events- A Coronary Angioscopic Study. Circ J 2018; 82: 2299-304.

12. Watanabe Y, Sakakura K, Taniguchi Y, et al. Determinants of slow flow following stent implantation in intravascular ultrasound-guided primary percutaneous coronary intervention. Heart Vessels 2018; 33: 226-38.

13. Kasahara T, Sakakura K, Yamamoto K, et al. Clinical Factors Associated with In-Hospital Mortality in Patients with Acute Myocardial Infarction Who Required Intra-Aortic Balloon Pumping. Int Heart J 2020; 61: 209-14.

14. Matsuo S, Imai E, Horio M, et al. Revised equations for estimated GFR from serum creatinine in Japan. Am J Kidney Dis 2009; 53: 982-92.

15. Mintz GS, Popma JJ, Pichard AD, et al. Patterns of calcification in coronary artery disease. A statistical analysis of intravascular ultrasound and coronary angiography in 1155 lesions. Circulation 1995; 91: 1959-65.

16. Teramoto $\mathrm{T}$, Tsuchikane $\mathrm{E}$, Matsuo $\mathrm{H}$, et al. Initial success rate of percutaneous coronary intervention for chronic total occlusion in a native coronary artery is decreased in patients who underwent previous coronary artery bypass graft surgery. JACC Cardiovasc Interv 2014; 7: 39-46.

17. Cutlip DE, Windecker S, Mehran R, et al. Clinical end points in coronary stent trials: a case for standardized definitions. Circu- lation 2007; 115: 2344-51.

18. Mauri L, Hsieh WH, Massaro JM, Ho KK, D'Agostino R, Cutlip DE. Stent thrombosis in randomized clinical trials of drugeluting stents. N Engl J Med 2007; 356: 1020-9.

19. Estrada JR, Paul JD, Shah AP, Nathan S. Overview of Technical and Cost Considerations in Complex Percutaneous Coronary Intervention. Interv Cardiol 2014; 9: 17-22.

20. Wilensky RL, Selzer F, Johnston J, et al. Relation of percutaneous coronary intervention of complex lesions to clinical outcomes (from the NHLBI Dynamic Registry). Am J Cardiol 2002; 90: 216-21.

21. Giustino G, Chieffo A, Palmerini T, et al. Efficacy and Safety of Dual Antiplatelet Therapy After Complex PCI. J Am Coll Cardiol 2016; 68: 1851-64.

22. Kirtane AJ, Doshi D, Leon MB, et al. Treatment of Higher-Risk Patients With an Indication for Revascularization: Evolution Within the Field of Contemporary Percutaneous Coronary Intervention. Circulation 2016; 134: 422-31.

23. Gaku N, Kengo T, Aoki J, et al. Clinical and angiographic outcomes of sirolimus-eluting stents implantation in Japanese patients in daily practice. Circ J 2006; 70: 1367-71.

24. Kawaguchi R, Tsurugaya H, Hoshizaki H, Toyama T, Oshima S, Taniguchi K. Impact of lesion calcification on clinical and angiographic outcome after sirolimus-eluting stent implantation in real-world patients. Cardiovasc Revasc Med 2008; 9: 2-8.

25. Otsuka F, Cheng Q, Yahagi K, et al. Acute Thrombogenicity of a Durable Polymer Everolimus-Eluting Stent Relative to Contemporary Drug-Eluting Stents With Biodegradable Polymer Coatings Assessed Ex Vivo in a Swine Shunt Model. JACC Cardiovasc Interv 2015; 8: 1248-60.

26. Jinnouchi $\mathrm{H}$, Mori $\mathrm{H}$, Cheng Q, et al. Thromboresistance and functional healing in the COBRA PzF stent versus competitor DES: implications for dual antiplatelet therapy. EuroIntervention 2019; 15: e342-53.

27. Prati F, Romagnoli E, Gatto L, et al. Clinical Impact of Suboptimal Stenting and Residual Intrastent Plaque/Thrombus Protrusion in Patients With Acute Coronary Syndrome: The CLI-OPCI ACS Substudy (Centro per la Lotta Contro L'InfartoOptimization of Percutaneous Coronary Intervention in Acute Coronary Syndrome). Circ Cardiovasc Interv 2016; 9: e003726.

28. Moscucci M, Fox KA, Cannon CP, et al. Predictors of major bleeding in acute coronary syndromes: the Global Registry of Acute Coronary Events (GRACE). Eur Heart J 2003; 24: 181523.

29. Tamanaha Y, Sakakura K, Taniguchi Y, et al. Comparison of Postcatheterization Pseudoaneurysm between Brachial Access and Femoral Access. Int Heart J 2019; 60: 1030-6.

30. Peduzzi P, Concato J, Kemper E, Holford TR, Feinstein AR. A simulation study of the number of events per variable in logistic regression analysis. J Clin Epidemiol 1996; 49: 1373-9.

31. Stoltzfus JC. Logistic regression: a brief primer. Acad Emerg Med 2011; 18: 1099-104. 